\title{
Analysis and Countermeasures of Abnormal Operation of Unloading Circuit
}

\author{
Xue Mei, Lin Huili and Zheng Xiaoli \\ Department of mechanical and electrical engineering, Beijing Polytechnic, Beijing, 100176, China
}

\begin{abstract}
Taking the two position two way valve unloading circuit, accumulator unloading circuit, double pump unloading circuit, liquid control sequence valve unloading circuit as examples, the paper analyses the reason of abnormal operation of several hydraulic unloading circuit, puts forward concrete measures, which provides basis for design and operation of hydraulic system.
\end{abstract}

\section{Introduction}

The function of the unloading circuit is to keep the hydraulic pump in a state of no load operation. When the actuator works intermittently or stops working, all or part of the hydraulic oil output from the hydraulic pump flows back to the oil tank under very low pressure. ${ }^{[1]}$ Unloading of the hydraulic system reduces power loss, reduces equipment operating costs, prevents system heating, and extends the life of pumps and motors.

Commonly used unloading circuits are: unloading circuit with three-position change-over valve; unloading circuit with unloading valve; unloading circuit with accumulator; circuit with pressure compensation variable pump; unloading circuit with two-position two-way valve; unloading circuit with solenoid relief valve, etc. ${ }^{[2]}$

Each kind of unloading circuit has its application scope. If it is applied reasonably, the equipment will run smoothly. If it is not applied properly, the equipment will stop working. In serious cases, the system will be damaged and personal accidents may occur. This paper discusses the causes of abnormal operation of unloading circuit and puts forward improvement measures.

\section{Unloading circuit of two-position two- way valve}

Figure 1 shows the unloading circuit of a two-position two-way valve. Hydraulic pump is a high-pressure and large-flow fixed displacement pump. ${ }^{[3]}$ When the system works, the two-position two-way valve energizes and cuts off the oil line of the return tank at the outlet of the oil pump. The pressure oil from the hydraulic pump enters the system completely and the actuating element begins to work. When the actuating element stops working, the solenoid loses power and the oil from the pump flows back to the tank via the two-position two-way valve, thus unloading the hydraulic system is realized. However, when the system runs, it is found that when the twoposition two-way valve loses power, the system cannot completely unload and causes the system to heat up.

It is found through inspection that the size of the twoposition two-way valve is too small to flow the output flow of the hydraulic pump back to the tank, which causes the pressure at the outlet of the pump to increase. The oilhydraulic difference through the two-position two-way valve increases and the two-position two-way valve acts as a throttle. The hydraulic pump cannot completely unload and causes the system to heat up. The reason is that the unloading circuit of two-position two-way valve is only suitable for small flow systems with hydraulic pump flow less than $63 \mathrm{~L} / \mathrm{min}$. As shown in Figure 2, a twoposition two-way valve should be used to control the unloading circuit of the pilot relief valve in a high pressure and large flow system.

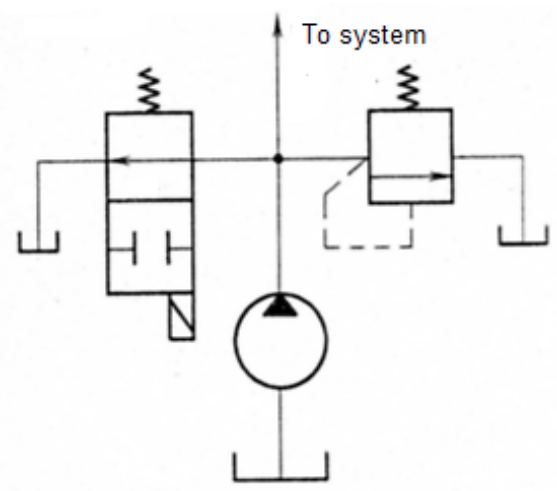

Fig. 1 unloading circuit of two position two way valve 


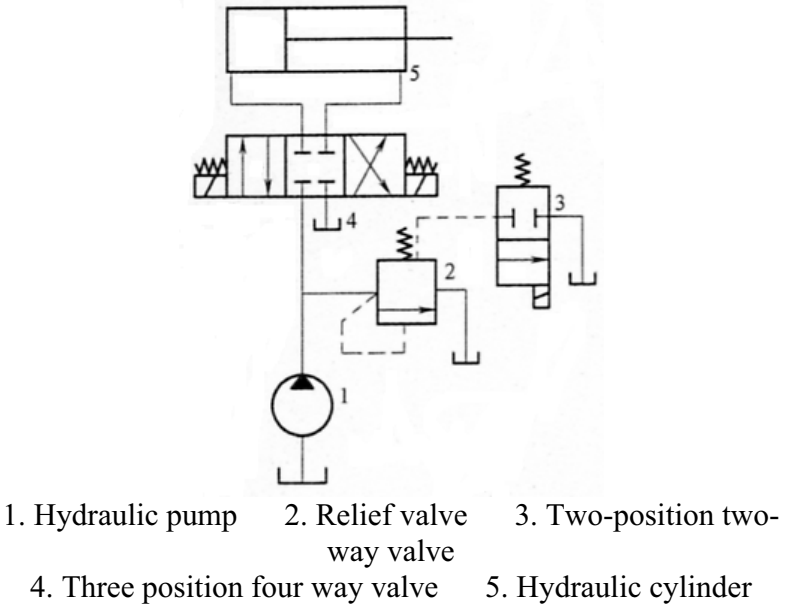

Fig. 2 unloading circuit of pilot relief valve controlled by twoposition two-way valve

In Figure 2, when the two-position two-way valve 3 is energized, the remote control port of the relief valve 2 leads to the tank, and the pressure oil from the pump 1 opens the relief valve 2 to flow back to the tank at a very low pressure, unloading the system. The unloading pressure is determined by the strength of the main valve spring of the relief valve and is generally $0.2 \sim 0.4 \mathrm{MPa}$. Valve 3 only passes through the hydraulic fluid from the control port of relief valve 2 , so valve 3 can select smaller valves and achieve remote control. However, when valve 3 is connected to the remote control port of valve 2 , the volume of control chamber of valve 2 increases and unstable phenomena may occur during operation.
Damping devices can be installed on the connected oil circuit at this time. This unloading circuit is suitable for high-flow hydraulic system.

\section{How to improve the pressure difference between the front and rear of speed control valve}

\subsection{Hydraulic circuit is not completely unloaded}

As shown in Figure 3 (a), when the pressure of accumulator 4 rises to the set pressure of unload valve 2 , valve 2 opens, oil pump 1 unloads, one-way valve 3 closes, and the system maintains pressure. When the system pressure is lower than the set pressure of valve 2, valve 2 closes and pump 1 refills the hydraulic system. At this time, the overflow valve 5 acts as a safety valve. The main failure of the hydraulic circuit is that the unloading is not complete and there is power loss. The reason is that when the pressure rises, unloading valve 2 is only partially opened to unload pump 1. ${ }^{[4]}$

Solution 1, as shown in Figure 3 (b), we can use small hydraulic control sequential valve 2 as the pilot valve to control the opening of main relief valve 5 , which can ensure full opening of valve 5 when unloading.

Solution 2, as shown in Figure 3 (c), the accumulator pressure opens the two-position three-way change-over valve 7 first, so that the two-position two-way changeover valve 6 is fully opened, thus the main relief valve 5 is fully opened and the pump 1 is fully unloaded.

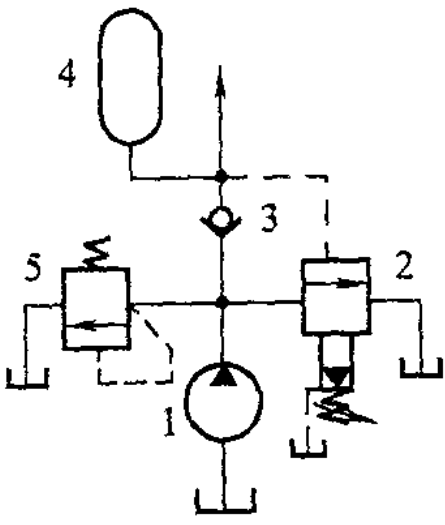

(a)

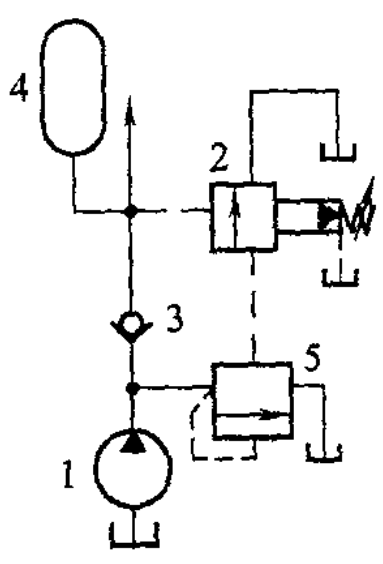

(b)

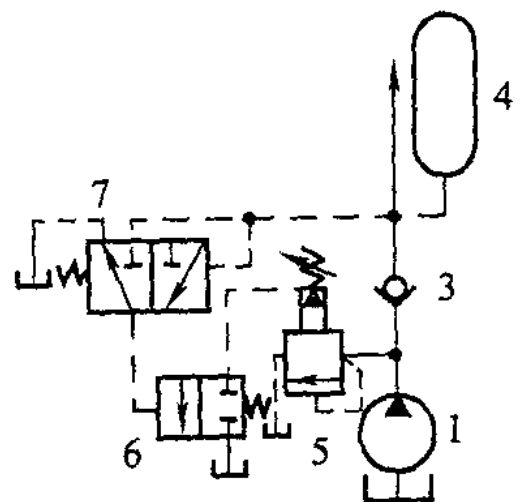

(c)
1. Hydraulic pump
2. Sequence valve
3. Check valve 4. Accumulator
5. Relief valve
6. Directional valve
7. Hydraulic directional valve

Fig. 3 unloading circuit of oil pump with accumulator maintaining pressure

\section{2 the unloading process of the system is unstable}

In the accumulator circuit shown in Fig. 4 (a), the pressure relay 3 controls the unloading or working of the hydraulic pump. During the operation of this circuit, the frequent alternation of "unloading working unloading" of the hydraulic pump is easy to occur, which makes the operation of the pump and valve extremely unstable and greatly shortens the service life of the hydraulic pump. The reason is that the system pressure fluctuates back and forth near the pressure value set by pressure relay 3 , causing the pressure relay to turn on and off repeatedly, thus causing the system to repeatedly unload and work. 


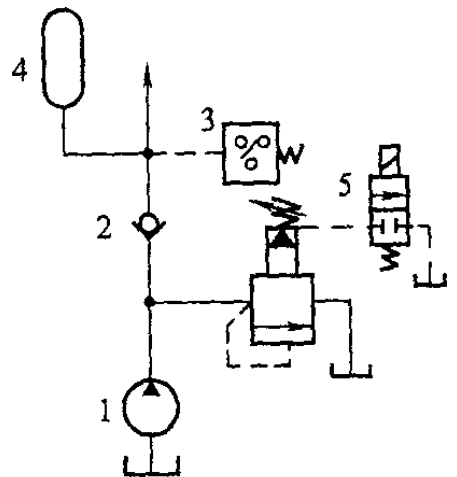

(a)

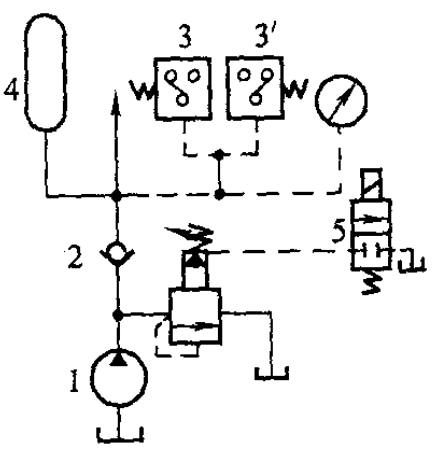

(b)

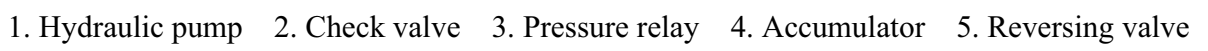

Fig. 4 unloading circuit of accumulator, pressure relay and electromagnetic relief valve

The solution is to use the double pressure relay shown in Figure 4(b) for differential pressure control ${ }^{[5]}$. Pressure relay 3 and 3 'are adjusted to high and low pressure respectively, unloading of hydraulic pump is controlled by high pressure, and pump re-operation is controlled by low pressure. Thus, when pump 1 is unloaded, the accumulator continues to drain oil until the pressure gradually drops below the low pressure setting value, the hydraulic pump will not be restarted. This process lasts for a while and prevents frequent switching of hydraulic pumps.

\section{Unloading circuit for double pump oil supply}

As shown in Fig. 5. During the operation of machine tools, steel rolling and other equipment, large flow and high speed work are required for the cylinder, which is supplied simultaneously by two pumps during fast stroke (e.g. feed of workpiece of machine tool).During the working stroke, the oil pressure rises, the unloading valve opens, the low pressure large flow pump 2 unloads, and the high pressure small flow pump 1 supplies oil.

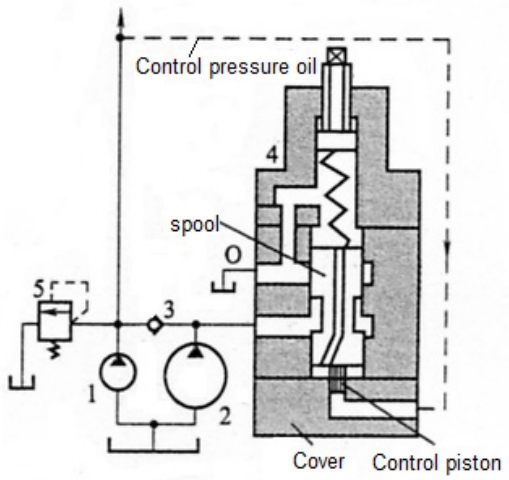

(b)

1. High pressure small flow pump 2. Low pressure large flow pump
$\begin{array}{ll}\text { 3. One-way valve } 4 \text {. Unloading valve } 5 \text {. Overflow valve }\end{array}$

Fig. 5 Unloading circuit of double pump oil supply

The following faults often occur in operation.

(1) The motor is heated seriously or even burnt out. The reason is that when the oil is supplied only by the high-pressure small flow pump 1, the check valve 3 is not closed well, the high-pressure oil is poured back, the load is heavy, and the motor is overloaded and heated, even burnt out.

(2) The system pressure cannot rise to the maximum. The reason is that the check valve 3 is not closed properly, and the control piston of unloading valve 4 is worn. The control pressure oil enters the lower chamber of the main spool of valve 4 through the clearance of the control piston outer diameter. Pushing the spool upwards opens the passage between the outlet of pump 2 and the return port. When the dual pumps supply oil, the pressure does not go up. Replacing the control piston can solve this problem, as shown in Fig. 5(b).

\section{Unloading circuit of hydraulic control sequential valve}

A machine factory has a self-made equipment. The actuating mechanism of the hydraulic equipment has the characteristics of long intermittent time and high speed of movement. It uses accumulator as auxiliary power source and cooperates with small flow hydraulic pump to realize fast movement of hydraulic cylinder. The equipment is unloaded by a hydraulic control sequential valve. The hydraulic circuit is shown in Figure 6(a). When the 
hydraulic system stops working, we switch the manual three-position four-way valve to the middle position. The hydraulic pump charges the accumulator with pressure oil. When the pressure inside the accumulator reaches the specified value, the hydraulic control sequence valve opens and the hydraulic pump unloads.

During the operation of the hydraulic equipment, the pressure at the outlet of the hydraulic pump fluctuates greatly and cannot be unloaded continuously and smoothly.

It is found that the hydraulic system has a long working interval. In order to achieve the continuous unloading of the hydraulic pump, the pressure oil in the accumulator must flow through the valve port of the hydraulic control sequential valve continuously. Because of the leakage of hydraulic components in the system, the accumulator pressure gradually decreases. When the pressure is lower than the opening pressure of the sequential valve, the sequential valve closes, the system stops unloading, and the hydraulic pump re-fills the accumulator with pressure oil; when the pressure rises to the opening pressure of the sequential valve, the sequential valve opens again and the hydraulic pump unloads again. In this way, the hydraulic pump is in the alternating state of unloading and filling, the power loss of the system is large, and the oil temperature increases, which greatly shortens the life of the hydraulic pump.

The modified circuit is shown in Figure $6(\mathrm{~b})$. The hydraulic circuit can operate normally by replacing the valves 2 and 3 in Figure 6(a) with the pilot unloading relief valve. As can be seen in Figure 6(b), the pilot-operated unloading relief valve consists of a main valve, a pilot valve and a check valve. During unloading, the pressure oil in the accumulator exerts an additional thrust on the pilot valve spool through the control plunger, thus ensuring the smooth unloading path of the pump. The oneway valve closes to maintain the accumulator pressure. Even if there is leakage in the circuit, the accumulator pressure decreases continuously, the hydraulic pump can maintain a long continuous unloading state. This example illustrates that pilot-operated unloading relief valves should be used instead of liquid-controlled sequential valves in long-term unloading hydraulic equipment.

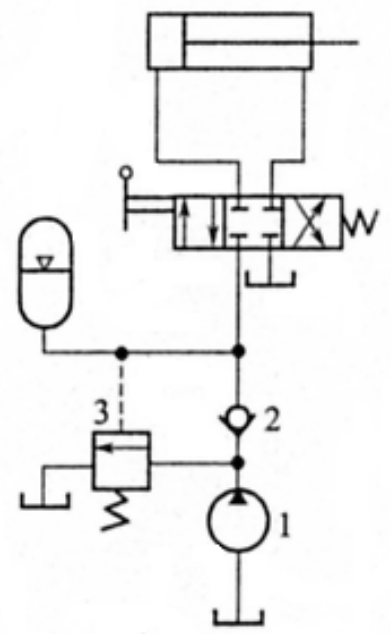

(a)

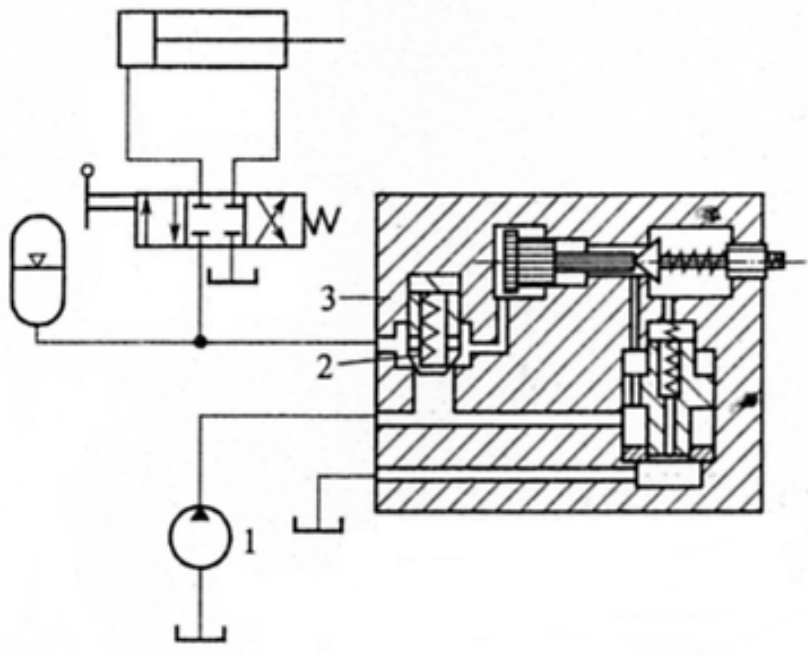

(b)

1. Hydraulic pump 2. One-way valve 3. Unloading valve

Fig. 6 Hydraulic system for continuous unloading

\section{Conclusions}

Various faults may occur in the operation of unloading circuit of hydraulic system. There are many reasons for the faults. According to different situations on site, the unloading elements and unloading circuit should be selected reasonably to avoid unstable unloading, incomplete unloading, repeated unloading, large unloading loss, hydraulic shock, lag of pressure rise, failure of unloading valve commutation, etc. We should give full play to the function of unloading circuit and improve the operation efficiency of hydraulic system.

\section{References}

1. Ma, Z.F. (2009) Hydraulics and Pneumatics. Mechanical Industry Publishing, Beijing.
2. Wang, J.W., Zhang, H.J., Huang, Y. (2005) Hydraulics and Pneumatics. Mechanical Industry Publishing, Beijing.

3. Cheng, H.M. (2006) Design and operation of unloading circuit. Coal mining machinery, Commun,6: 948-950.

4. Zhao, Y.Y.(1999) Hydraulic control valve and its repair. Shanghai Jiao Tong University Publishing, Shanghai.

5. Zhang, Y.L. (2008) Questions and Answers on Hydraulic Maintenance Technology. Chemical Industry Publishing, Beijing. 